\title{
G-DRAZIN INVERSES FOR OPERATOR MATRICES
}

\author{
Huanyin Chen and Marjan Sheibani Abdolyousefi
}

\begin{abstract}
Additive results for the generalized Drazin inverse of Banach space operators are presented. Suppose the bounded linear operators $a$ and $b$ on an arbitrary complex Banach space have generalized Drazin inverses. If $b^{\pi} a b a=0$ and $a b^{2}=0$, then $a+b$ has generalized Drazin inverse. This extends the main results of Djordjević and Wei (J. Austral. Math. J., 73(2002), $115-125$ ). Then we apply our results to $2 \times 2$ operator matrices and thereby generalize the results of Deng, Cvetković-Ilić and Wei (Linear and Multilinear Algebra, 58(2010), 503-521).
\end{abstract}

Mathematics subject classification (2010): 15A09, 32A65, 16E50.

Keywords and phrases: g-Drazin inverse, additive property, operator matrix, spectral idempotent.

\section{REFERENCES}

[1] S. L. Campbell and C. D. Mayer, Generalized Inverse of Linear Transformations, Pitman, London, 1979.

[2] S. L. CAMPBelL, The Drazin inverse and systems of second order linear differential equations, Linear and Multilinear Algebra 14(1983), 195-198.

[3] N. Castro-González and J.J. Koliha, New additive results for the g-Drazin inverse, Proceedings of the Royal Society of Edinburgh, Section A 134(2004), 1085-1097.

[4] X. Chen And R. E. Hartwig, The group inverse of the triangular matrix, Linear Algebra Appl., 237-238(1996), 97-108.

[5] C. Deng, D. S. Cvetković-Ilić And Y. WeI, Some results on the genrealized Derazin inverse of operator matrices, Linear and Multilinear Algebra 58(2010), 503-521.

[6] C. Deng And Y. WeI, New additive results for the generalized Drazin inverse, J. Math. Analysis Appl., 379(2010), 313-321.

[7] D.S. DJordjević AND Y. WeI, Additive results for the generalized Drazin inverse, J. Austral. Math. Soc., 73(2002), 115-125.

[8] L. Guo, Representations of Generalized Drazin Inverses for Operator Matrices, Ph.D. Thesis, Jilin University, Changchun, 2010.

[9] L. Guo, J. CHEN AND H. Zou, Some additive results for the generalized Drazin inverse in a Banach algebra, J. Southeast Univ., 33(2017), 382-386.

[10] Y. JIANG, Y. WEN AND Q. ZENG, Generalizations of Cline's formula for three generalized inverses, Revista. Un. Mat. Argentina, 58(2017), 127-134.

[11] Y. Liao, J. Chen And J. Cui, Cline's formula for the generalized Drazin inverse, Bull. Malays. Math. Sci. Soc., 37(2014), 37-42.

[12] V.G. Miller And H. Zguitti, New extensions of Jacobson's lemma and Cline's formula, Rend. Circ. Mat. Palermo, II. Ser., Published online: 09 February 2017, Doi: 10.1007/s12215-017-0298-6.

[13] D. Mosić, Extensions of Jacobson's lemma for Drazin inverses, Aequat. Math., Published online: 04 April 2017, Doi: 10.1007/s00010-017-0476-9.

[14] Y. Wei And H. DiAO, On group inverse of singular Toeplitz matrices, Linear Algebra Appl., 339(2005), 109-123.

[15] H. YANG AND X. LiU, The Drazin inverse of the sum of two matrices and its applications, J. Comput. Appl. Math., 235(2011), 1412-1417. 